He had no additional neurological symptoms during the next 13 months. $\mathrm{He}$ has stopped smoking and drinking alcohol Arterial blood pressure and the lipid profile have returned to normal.

Leiguarda et $a l^{1}$ reported four patients with frontal and parietal lesions in which abnormal complex movements akin to the alien hand syndrome were present in brief episodes, sometimes followed by focal and generalised seizures. Our patient was very different, having multiple risk factors for stroke, Leriche syndrome, and three previous cerebrovascular lesions. The described episode had an abrupt onset and gradua recovery, featuring some of the most typica symptoms of reversible ischaemic dysfunction.

The pattern of abnormal movements and the pronounced leg weakness in our patient is suggestive of damage to the medial frontal lobe. $^{23}$ The typical syndrome after midline lesions has a different presentation, with much intermanual conflict, ${ }^{3}$ the abnorma behaviour of the affected hand always being triggered by volitional movements of the normal hand. ${ }^{4}$ Sensory changes have already been described in patients with alien hand syndrome. ${ }^{15} \mathrm{We}$ speculate whether the ipsilateral hypaesthesia (thalamic?) somehow contributed to the pattern of abnormal movements of the arm, as in patients with parieto- occipital lesions. ${ }^{5}$

Reversible reduction of blood flow to the medial frontal cortex responsible for the formation of motor programmes (plus thalamoparietal region?) should now be regarded as another cause for the transient alien hand phenomenon.

We thank Dr Thomas R Price of the Departmen of Neurology of the University of Maryland School of Medicine for his critical review of the manuscript.

CHARLES ANDRÉ

Cerebrovascular Diseases Section Department of Neurology, Clementino Fraga Filho University Hospital, Federal University of Rio de Faneiro, Brazil. RM-Magnetic Resonance Center Rio de faneiro, Brazi

Correspondence to: Dr Charles André, Serviço de Neurologia, Hospital Universitário Clementino Fraga Filho, Av Brigadeiro Trompowsky, $s / n^{\circ}, 10^{\circ}$ andar CEP 21941-590, Ilha do Fundão, Rio de Janeiro, RJ-Brasil

1 Leiguarda R, Starkstein S, Nogués M, Berthier M, Arbelaiz R. Paroxismal alien hand syndrome. F Neurol Neurosurg Psychiatry 1993; 56:788-92.

2 McNabb AW, Carroll WM, Mastaglia FL "Alien hand" and loss of bimanual coordination after dominant anterior cerebral artery territory infarction. $f$ Neurol Neurosurg territory infarction. $\mathcal{F}^{\mathrm{f}} \mathrm{N}$

3 Goldberg G, Mayer NH, Toglia JU. Medial frontal cortex infarction and the alien hand sign. Arch Neurol 1981;38:683-6.

4 Wilson DH, Reeves A, Gazzaniga $M$, et al. Cerebral commissurotomy for control of intractable seizures. Neurology 1977;27: 708-15.

5 Levine DN, Rinn WE. Opticosensory ataxia and alien hand syndrome after posterior cerebral artery territory infarction. Neurology 1986;36:1094-7.

\section{Brain water intoxication and CT hypolucencies in ovarian hyperstimula- tion syndrome complicating gametes intrafallopian transfer (GIFT) and tubal pregnancy}

The ovarian hyperstimulation syndrome is a recognised complication of hormone treatment for assisted conception, and includes serositis with ascites and hydrothorax in its most severe form. ${ }^{1}$ Our patient had probable syndrome of inappropriate antidiuretic hormone secretion (SIADH) complicating ovarian hyperstimulation syndrome and ectopic (tubal) pregnancy. The patient was a 36 year old university educated bank clerk with ampullary tubal occlusion. Four weeks after gametes intrafallopian transfer (GIFT) procedures and hormonal treatment with FSH (150 UI for 10 days) and GnRH (10 000 UI) pregnancy tests were positive and the patient developed serohaematic ascites, bilateral hydrothorax, and severe orthopnoea with tachypnoea and hypotension, due to severe ovarian hyperstimulation syndrome. She underwent laparotomy; a tubal pregnancy was recognised and aborted. She was then transferred to the intensive care unit and treated with blood transfusion (red cell were $1880000 / \mathrm{ml}$ ), methylprednisone, glucosate, and isotonic saline solutions. Blood pressure was maintained with dopamine. Concentrations of $\mathrm{Na}^{+}$were $133-147 \mathrm{mEq} / \mathrm{l}$ for four days, during which time liquid balance was negative $(-300$ to $-600 \mathrm{ml}$ ), creatinine clearance was normal, serum creatinine was $0.7 \mathrm{mg} / \mathrm{dl}$, blood urea nitrogen $11 \mathrm{mg} / \mathrm{dl}$, serum osmolarity $327 \mathrm{mmol} / 1$, urinary osmolarity $630 \mathrm{mmol} / \mathrm{l}$, and urinary specific gravity 1017 . On the fifth day she had two tonic-clonic seizures treated with diazepam . She became confused and lethargic; $\mathrm{Na}^{+}$concentrations were $134 \mathrm{mEq} / 1$ two hours before the seizures and $118 \mathrm{mEq} / \mathrm{l}$ one hour after. Fluid balance was $1000 \mathrm{ml}$ positive. On the same day creatinine clearance

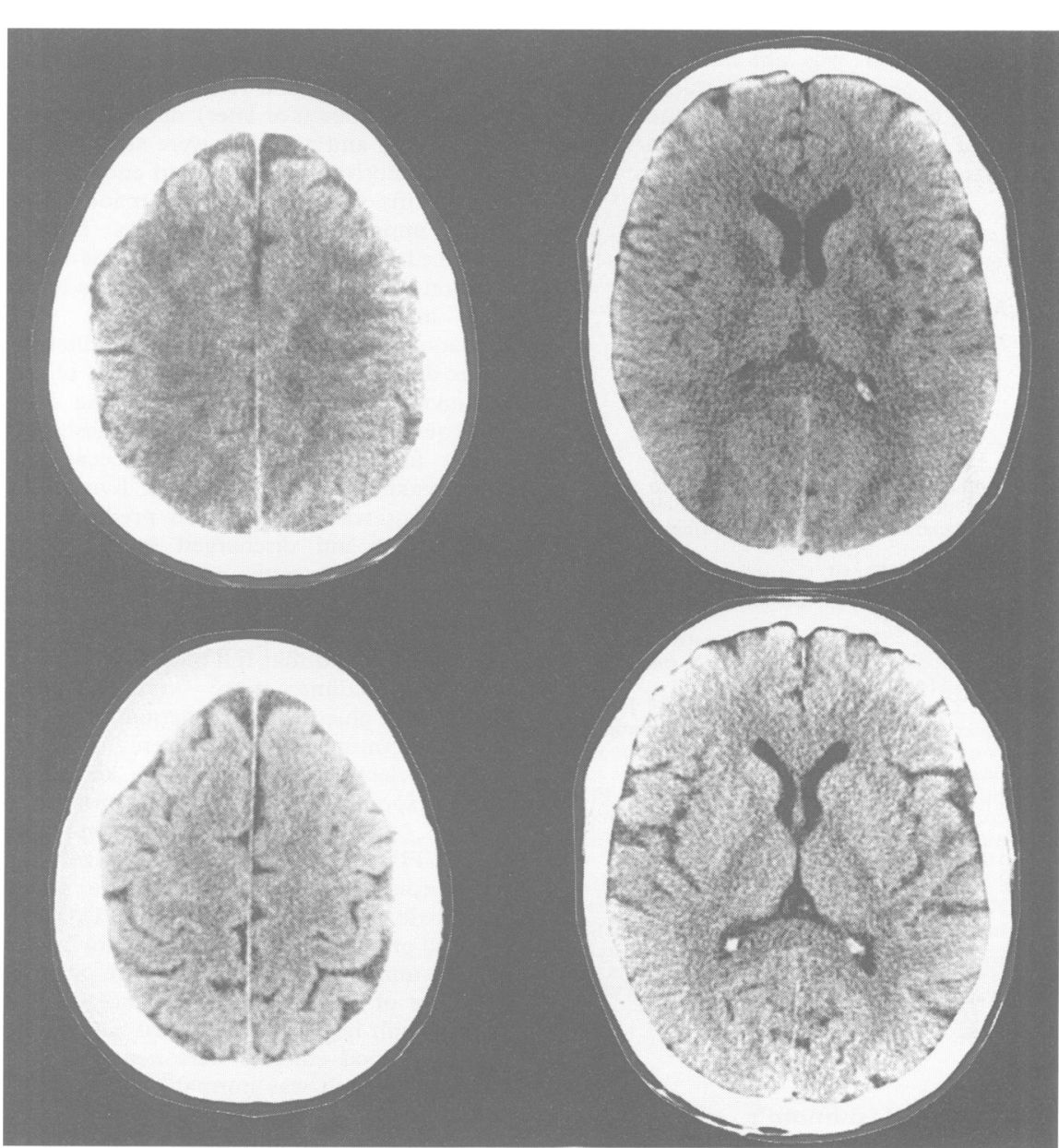

was normal, serum osmolarity was 306 $\mathrm{mmol} / \mathrm{l}$, urinary osmolarity $807 \mathrm{mmol} / \mathrm{l}$, urinary specific gravity $1038.30 \mathrm{mEq} / \mathrm{l} \mathrm{Na}+$ were given with demeclocycline and furosemide.

A CT performed on the same day was normal. Concentrations of $\mathrm{Na}^{+}$increased to $130 \mathrm{mEq} / \mathrm{l}$ in the next 12 hours, but she became stuporous, then comatose (Glasgow coma scale 4; pupils $2 \mathrm{~mm}$; presence of light reflex). An EEG showed diffuse $2-4 \mathrm{~Hz}$ slowing. Creatinine clearance was again normal, serum osmolarity was $285 \mathrm{mmol} / \mathrm{l}$, urinary osmolarity $860 \mathrm{mmol} / 1$, and urinary specific gravity 1039. Parenteral phenobarbitone ( $3 \mathrm{mg} / \mathrm{kg} /$ day) was added to current treatment, including albumin, demeclocycline, furosemide, and isotonic solution to increase $\mathrm{Na}^{+}$concentration at a rate of less than $5 \mathrm{mEq} / \mathrm{l} /$ day. Concentrations of $\mathrm{Na}^{+}$ were $131-137 \mathrm{mEq} / 1$ in the next five days, liquid balance was positive $(+950 \mathrm{ml}$ to $+600 \mathrm{ml}$ ) until the 10 th day, when another seizure (one minute duration) occurred. Intracranial pressure was $32 \mathrm{ml} \mathrm{H} \mathrm{H}_{2} \mathrm{O}$ CSF was normal. Creatinine clearance was normal, serum osmolarity $325 \mathrm{mmol} / 1$, urinary osmolarity $800 \mathrm{mmol} / \mathrm{l}$, and urinary specific gravity 1030 . A CT performed on the same day showed non-homogeneous patchy areas of hypolucency (fig 1); her liquid balance became negative $(-1200 \mathrm{ml})$ and remained so for the next 10 days $(-1400 \mathrm{ml}$ to -300 $\mathrm{ml}$ ). Consciousness progressively improved; the patchy hypolucencies were not present on $\mathrm{CT}$ on the 17th day after admission to intensive care (figure). On the 20th day she

Top row: when the patient was comatose, five days after the first seizure, patchy areas of nonhomogeneous hypolucencies are present, prominently in the right dorsal prefrontal, left central parietal, right parieto-occipital, right internal, and left external capsule. Bottom row: control CT 30 days later, when the patient was fully alert; no hypolucencies are present. 
was fully alert. Serum and urinary osmolarity became normal, urinary specific gravity was 1005-1025. She recovered from ovarian hyperstimulation syndrome and laparotomy during the next month.

Brain MRI and CT performed during the next five years were normal, as were repeated neurological and psychiatric examinations. The patient's IQ was 126-130.

Severe serositis with ascites and hydrothorax due to ovarian hyperstimulation syndrome syndrome and haemoperitoneum due to tubal pregnancy, with hypovolaemia, anaemia, and hyposmolar serum concentrations masked an SIADH that was heralded by seizures, followed by a prolonged lethargic state. Collateral evidence of SIADH was obtained by normal creatinine clearance ${ }^{2}$ with urine hyperconcentration. The symptoms of CNS water intoxication, as usual, ${ }^{23}$ appeared during a sudden decrease in $\mathrm{Na}^{+}$ serum concentration, and were treated slowly to avoid central pontine myelinolisis. During SIADH, CT showed several patchy areas of hypolucency, resembling severe lesions of acute hypoxic-ischaemic encephalopathy with brain oedema. ${ }^{45}$ Hypoxicanoxic lesions are, however, usually caused by residual neurological or psychiatric deficit, and CT shows evolution of lesions, with ventricular enlargement and leucomalacy. ${ }^{45}$ In this patient instead the hypolucencies disappeared, the patient had no neurological or psychiatric alterations, and later CT and MRI did not show residual areas of altered signal corresponding to early hypolucencies. Furthermore, unlike the situation in hypoxic-anoxic lesions, ${ }^{45}$ the basal ganglia did not seem to be involved, and the ventricular system was not narrowed as in severe brain oedema. We concluded therefore that water intoxication induced $C T$ images of patchy hypolucencies rather than the expected homogeneous hypolucency. ASTRID THOMAS DONATO MELCHIONDA TOMMASO FULGENTE Department of Neurology,

Institute of Neurology and Behavioural Sciences, State University of Chieti, Chieti, Italy AMEDEO COSTANTINI MICHELE SCESI Intensive Care Unit,

State University of Chieti, Chieti, Italy

Correspondence to: Professor Marco Onofri, Clinica Neurologica, Ospedale ex Pediatrico, Via Martiri Lancianesi 6, 66100 Chieti, Italy.

1 Schenker JG, Ezra J. Complications of assisted reproductive techniques. Fertil Steril 1994;3: 411-22.

2 Narins RG. Therapy of hyponatremia: Does haste make waste? [editorial] $N$ Engl $\mathcal{F}$ Med 1986;314:1573-5

3 Bleck TP, Smith MC, Pierre-Louis SJ, Jares JJ, Murray J, Hansen CA. Neurologic complications of critical medical illnesses. Crit Care Med, 1993;21:98-103

4 Tippin J, Adams HP, Smoker WRK. Early computed tomographic abnormalities following profound cerebral hypoxia. Arch Neurol 1984;41:1098.

5 De Reuck J, Decoo D, Vienne J, Strijckmans $\mathrm{K}$, Lehmahieu I. Significance of white matter K, Lehmahieu I. Significance of white matter lucencies in posthypoxic-ischemic encephalopathy: comparison of clinical status and of computed and positron emission tomo-
graphic findings. Eur Neurol 1992;32:334-9.

\section{Thyrotoxic Hashimoto's encephalopa- thy}

Thyroid disease is associated with several neurological disorders, ${ }^{1}$ of which one of the rarest and least well understood is
Hashimoto's encephalopathy. This was originally postulated to be a distinct disease entity by Brain et al in $1966^{2}$ and there have subsequently been case reports substantiating the hypothesis that it represents a unique condition. ${ }^{3-5}$ The characteristic features are a subacute onset of confusion with altered consciousness, seizures, and stroke-like events that respond to steroids and which occur in the context of high anti-microsomal antibody titres. ${ }^{3}$ To date all the patients reported have been either euthyroid or hypothyroid at the time of presentation. We present a patient with Hashimoto's encephalopathy with pronounced thyrotoxicosis, that was successfully managed with steroids, carbimazole, and propanolol.

A 49 year old woman presented with a six month history of weight loss and a three month history of proximal arm pain and hand tremor. Two weeks before admission she developed a progressive left sided weakness involving the arm and leg in conjunction with a left hemianaesthesia. On examination at admission she was flushed, feverish, and tachycardic with a hyperdynamic circulation. Her thyroid gland was slightly enlarged but there was no associated bruit. Cranial nerve examination disclosed left visual inattention as the only abnormality. Limb examination showed a moderately severe left hemiparesis with left sensory inattention, generalised hypereflexia, and bilaterally extensor plantar responses. She had wasting of the shoulder girdle muscles and adhesive capsulitis of the shoulder joints bilaterally. In the days immediately after admission she became drowsy, confused, and had florid visual hallucinations, while independently having runs of paroxysmal atrial fibrillation. As a result of the original negative findings (see later) dexamethasone $(12 \mathrm{mg} /$ day) and acyclovir were started with the presumptive diagnosis of an encephalitis or vasculitis. On this regime she made a dramatic improvement, which was further enhanced by the treatment of her thyrotoxicosis on receipt of her thyroid function tests. The introduction of carbimazole and propanolol was then followed by a reduction in the dexamethasone and a cessation of the acyclovir. Attempted steroid weaning over subsequent days provoked a recrudescence of her focal symptoms on two occasions, with weakness of her right arm. Eventually the patient was stabilised on prednisolone $(40 \mathrm{mg} /$ day $)$ and discharged on a slowly reducing course with no relapses three months after discharge.

Investigations performed during her inpatient stay showed that full blood count, erythrocite sedimentation rate, urea, electrolytes, glucose, liver function tests, and serum immunoglobulins were normal. Protein electrophoresis showed an acute phase response with a $\mathrm{C}$ reactive protein of $32 \mathrm{mg} / \mathrm{l}$. Her autoantibody screen and VDRL/TPHA serology were negative, but her thyroid function tests showed her to be thyrotoxic with TSH less than $0.03 \mathrm{U} / 1$, free T4 $>80 \mathrm{pmol} / 1$, and free T3 $41 \mathrm{pmol} / 1$. Her thyroid microsomal antibodies were positive at a titre of 1:6400. Her CSF analysis was normal with negative oligoclonal bands and repeated blood cultures were negative. Her chest radiograph was normal but her ECG showed a sinus tachycardia with episodes of paroxysmal atrial fibrillation. Her EEG showed occasional brief bursts of frontal slow activity which spread posteriorly and brain CT with contrast and MRI with gadolinium were normal. In addition a transthoracic and transoesophageal ECG along with MRI of her heart were all normal

These results show that she had a pronounced thyrotoxicosis with antimicrosomal antibodies. There was no evidence for any fixed structural lesion within the CNS accounting for her neurological condition as evidenced by her normal brain CT and MRI.

Autoimmune thyroid disease can be considered as a range of clinical disorders reflecting the variety of autoantibodies present. Hashimoto's disease is characterised by the presence of thyroid antimicrosomal antibodies and has rarely been associated with an encephalopathic process of unknown aetiology. All previously described patients have either been euthyroid or hypothyroid and this is the first description of an encephalopathy in combination with thyrotoxicosis. As the mechanism of encephalopathy is uncertain the term thyroid related encephalopathy is preferable. Although atria fibrillation was present in our patient, the normal heart and head imaging argues gainst an embolic cause for her condition. Furthermore, her remarkable steroid responsiveness suggests an autoimmune cause for her fluctuating multifocal encephalopathy.

Various mechanisms have been postulated to account for this unusual condition. One possibility is demyelination, which can virtually be discounted on the basis of our results as both MRI and CSF were normal. More likely explanations are either a multifoca abnormality of cerebral perfusion or a patchy defect of metabolism.

This patient completes the repertoire of thyroid states seen in thyroid related encephalopathies and emphasises the need to assess thyroid function and autoantibody tatus in patients presenting with encephalopathy and stroke-like events in the absence of structural or infective aetiologies. ROGER BARKER JOHN ZAJICEK Department of Neurology Addenbrooke's Hospital Cambridge CB2 6SP, UK

Correspondence to: Dr Roger Barker, National Hospital for Neurology and Neurosurgery, Quee Hospital for Neurology and Neuro
Square, London WCIN 3BG, UK.

1 Thrush DC, Boddie HG. Episodic encephalopathy associated with thyroid disorders. If Neurol Neurosurg Psychiatry 1974 37:696-700.

2 Lord Brain, Jellinek EH, Ball K. Hashimoto' disease and encephalopathy. Lancet 1966;ii: 512-4.

3 Shaw PJ, Walls TJ, Newman PK, Cleland PG, Cartlidge NEF. Hashimoto's encephalopathy: A steroid-responsive disorder associated with high anti-thyroid antibody titers-report of 5 cases. Neurology 1991;41:228-33.

4 Ishii K, Hayashi A, Tamaoka A, Mizusawa H, Shoji S. A case of Hashimoto's encephalopathy with a relapsing course related to the menstrual cycle. Clinical Neurology 1993;33: 995-7.

5 Shein M, Apter A, Dickerman Z, Tyano S, Gadoth N. Encephalopathy in compensated Hashimoto thyroiditis: A clinical expression of autoimmune cerebral vasculitis. Brain Dev 1986;8:60-4.

\section{Cerebral salt wasting syndrome}

Excessive natriuresis, resulting in hyponatraemia and polyuria, is an often recognised complication after subarachnoid haemorrhage. Initially this was attributed to inappropriate antidiuretic hormone ( $\mathrm{ADH})$ secretion resulting in water retention, but 